\title{
Towards Bridging Translational Gap in Cardiotoxicity Prediction: an Application of Progressive Cardiac Risk Assessment Strategy in TdP Risk Assessment of Moxifloxacin
}

\author{
Nikunjkumar Patel, ${ }^{1,2,5}$ Oliver Hatley, ${ }^{1}$ Alexander Berg, ${ }^{3}$ Klaus Romero, ${ }^{3}$ Barbara Wisniowska, ${ }^{2}$ Debra Hanna, ${ }^{3}$ \\ David Hermann, ${ }^{4}$ and Sebastian Polak ${ }^{1,2}$
}

Received 30 November 2017; accepted 31 January 2018; published online 14 March 2018

\begin{abstract}
Drug-induced cardiac arrhythmia, especially occurrence of torsade de pointes (TdP), has been a leading cause of attrition and post-approval re-labeling and withdrawal of many drugs. TdP is a multifactorial event, reflecting more than just drug-induced cardiac ion channel inhibition and QT interval prolongation. This presents a translational gap in extrapolating pre-clinical and clinical cardiac safety assessment to estimate TdP risk reliably, especially when the drug of interest is used in combination with other QT-prolonging drugs for treatment of diseases such as tuberculosis. A multi-scale mechanistic modeling framework consisting of physiologically based pharmacokinetics (PBPK) simulations of clinically relevant drug exposures combined with Quantitative Systems Toxicology (QST) models of cardiac electro-physiology could bridge this gap. We illustrate this PBPK-QST approach in cardiac risk assessment as exemplified by moxifloxacin, an anti-tuberculosis drug with abundant clinical cardiac safety data. PBPK simulations of moxifloxacin concentrations (systemic circulation and estimated in heart tissue) were linked with in vitro measurements of cardiac ion channel inhibition to predict the magnitude of QT prolongation in healthy individuals. Predictions closely reproduced the clinically observed QT interval prolongation, but no arrhythmia was observed, even at $\times 10$ exposure. However, the same exposure levels in presence of physiological risk factors, e.g., hypokalemia and tachycardia, led to arrhythmic event in simulations, consistent with reported moxifloxacin-related TdP events. Application of a progressive PBPK-QST cardiac risk assessment paradigm starting in early development could guide drug development decisions and later define a clinical "safe space" for postapproval risk management to identify high-risk clinical scenarios.
\end{abstract}

KEY WORDS: hERG; moxifloxacin; torsade de pointes; quantitative systems toxicology; QT prolongation.

\section{INTRODUCTION}

Cardiac toxicity is one of the leading causes of high attrition rate at various stages of drug development, withdrawal of several marketed drugs, and re-labelling of many

Electronic supplementary material The online version of this article (https://doi.org/10.1208/s12248-018-0199-4) contains supplementary material, which is available to authorized users.

\footnotetext{
${ }^{1}$ Simcyp Limited, a Certara Company, Blades Enterprise Centre, John Street, S2, 4SU, Sheffield, UK.

${ }^{2}$ Unit of Pharmacoepidemiology and Pharmacoeconomics, Faculty of Pharmacy, Jagiellonian University Medical College, Krakow, Poland.

${ }^{3}$ Critical Path Institute, Tucson, Arizona, USA.

${ }^{4}$ Certara USA, Inc, Princeton, New Jersey, USA.

${ }^{5}$ To whom correspondence should be addressed. (e-mail: Nikunjkumar.Patel@certara.com)
}

drugs (1-4). Among various types of cardiotoxic effects, arrhythmogenicity is the most common post-approval adverse event (3). Of particular concern is torsade de pointes (TdP), a life-threatening arrhythmia that can degenerate into ventricular fibrillation.

Due to the potentially significant safety impact and corresponding regulatory concern of drug-induced cardiotoxicity, careful assessment of pro-arrhythmic potential is an integral part of pre-clinical and clinical safety evaluation of an investigational new drug (IND). There are several methods available for cardiac risk assessment, including in silico, in vitro, and ex vivo models as well as in vivo studies in animals and humans. Selection of the appropriate model is dependent upon the stage of development and the cardiac safety questions relevant to each development program. The most commonly utilized models during pre-clinical development include in vitro ion current inhibition models, where the human ion channels involved in cardiac electrophysiology are 
heterologously expressed in non-human or human cell lines (5). These approaches continue to evolve, with newer approaches such as the Comprehensive in vitro Proarrhythmia Assay (CiPA) initiative, assessing the role of stem cell-derived cardiomyocytes in cardiac safety assessment (6-10).

Despite the availability of multiple models and the continued advances in cardiac risk assessment, the number of reports of TdP remains significant (over 100 cases of the TdP reported to the FDA Adverse Event Reporting System (FAERS) every year) and almost half of them are related to non-cardiovascular drugs $(4,11)$. This reflects a translational gap in the quantitative clinical extrapolation from the typical cardiac safety data collected, such as early non-clinical drug screens for cardiac ion channel inhibition, particularly hERG (human ether-a-go-go gene) inhibition, or the propensity for QT interval prolongation as assessed in early phase and thorough QT trials (TQT) clinical trials (12). Consequently, to minimize risk under an optimized drug development paradigm, early stage gates are often employed which only progress compounds predicted or observed to have little to no increase in QT interval, raising concerns that therapeutically promising agents may be triaged inappropriately. These concerns represent findings that the occurrence of TdP is multifactorial, reflecting more than just a drug's ability to inhibit hERG or increase the QT interval. These additional factors, such as gender, co-medications, comorbidities, and genetic polymorphisms, make a drug relatively safe for most people potentially cardiotoxic for a few individuals under specific circumstances (13).

Translational tools with improved predictive performance that account for the multifactorial determinants of cardiotoxicity, particularly $\mathrm{TdP}$, are needed to improve both the cardiac risk assessment and attrition rates of compounds in development. One such set of tools are physiologically based, biophysically detailed models of cardiac physiology that can characterize the contributions of multiple ion channel inhibition on the electrophysiology of human cardiomyocytes. These models can bridge the translational gap in cardiac safety assessment during the early drug development stage by faithfully reproducing the mechanistic exposure-response relationships from the molecular level to the full organism level. Specifically, simulations using this framework can link predicted or observed drug pharmacokinetics (PK) via physiologically based PK (PBPK) modeling with dynamic ion channel inhibition and subsequent effects on electrocardiography (ECG) parameters in virtual patients, which represent an array of physiologies. In addition to ECG metrics of interest (i.e., change in QTc interval) across a range of drug exposures, the derived ECG outputs can be directly utilized in the cardiac risk algorithm recently developed by Polak et al. (14). This algorithm, which can operate without well-characterized human PK and pharmacodynamics (PD) aspects, predicts the probability of a compound causing TdP at early stage of development when used as monotherapy in the context of the physiology of a generally healthy average human and improves upon earlier algorithms in that it considers potential supra-therapeutic concentrations as well as the impact of inhibition of multiple ion channels other than hERG in risk stratification.
The current work exemplifies a model-based quantitative systems toxicology (QST) strategy for progressive cardiac risk assessment, where the risk assessment for a novel compound is refined progressively as clinical knowledge is enriched through predict-learn-confirm cycles. The individual model components are iteratively verified/refined, to reduce uncertainty and increase confidence, making them adaptable to more adequately address questions around therapeutic performance, as they arise during clinical development. The Cardiac Risk Algorithm as proposed by Polak et al. is one component model that can be particularly informative during early phase development to estimate potential cardiac safety risk even in the absence of detailed information about human $\mathrm{PK}$ and PD, as sensitivity analyses may be performed to simulate a range of possible clinical scenarios. As more PK and PD information about the drug becomes available with further drug development, these initial predictions can be verified and refined, and ultimately may be superseded by a more detailed PBPK-QST model platform. Once sufficiently verified, the established and enriched model can be used to simulate clinical scenarios to evaluate effects of intrinsic and extrinsic factors (i.e., special populations, drug-drug interactions, co-morbidities) to establish the clinical cardiac risk scenarios (Fig. 1).

To illustrate this approach, a series of simulations were performed to reflect virtual administration of the antiinfective, moxifloxacin (MOXI). MOXI was chosen as an exemplar compound as it is the most commonly used positive control in TQT trials and thus has well-established human PK and PK-ECG profiles. Furthermore, MOXI is active against Mycobacterium tuberculosis (Mtb), the causative pathogen of tuberculosis (TB), and thus is administered as a component of anti-TB drug regimens. Cardiac risk assessment in TB is of specific importance, as many existing and emerging anti-TB agents have a known or suspected propensity for QT prolongation. As the application of a model-based QST strategy for progressive cardiac risk assessment could be advantageous in the prioritization of compounds for inclusion in anti-TB regimens, MOXI serves as an illustrative example of how this approach could be applied during pre-clinical and early clinical development. Here, we report a utilization of the cardiac QST models implemented within Cardiac Safety Simulator (CSS) platform (Certara, Sheffield, UK), previously known as Tox-Comp platform (15) for the quantitative prediction of QT prolongation in humans at therapeutic dose and the risk of causing TdP. We also assessed the impact of potential supra-therapeutic concentration of MOXI in causing $\mathrm{TdP}$ in silico as well as existing conditions such as hypokalemia which was not part of early-stage risk stratification algorithm.

\section{MATERIALS AND METHODS}

Simcyp Simulator V16 (Certara, Sheffield, UK) was used for PBPK simulations. The Advanced Dissolution, Absorption and Metabolism (ADAM) model was used to mechanistically simulate the formulation effects on drug absorption, and 14 organ full body perfusion limited PBPK distribution model was used to model disposition after systemic absorption $(16,17)$. The recently established PBPK model parameters (Supplement 1) were used to simulate the 


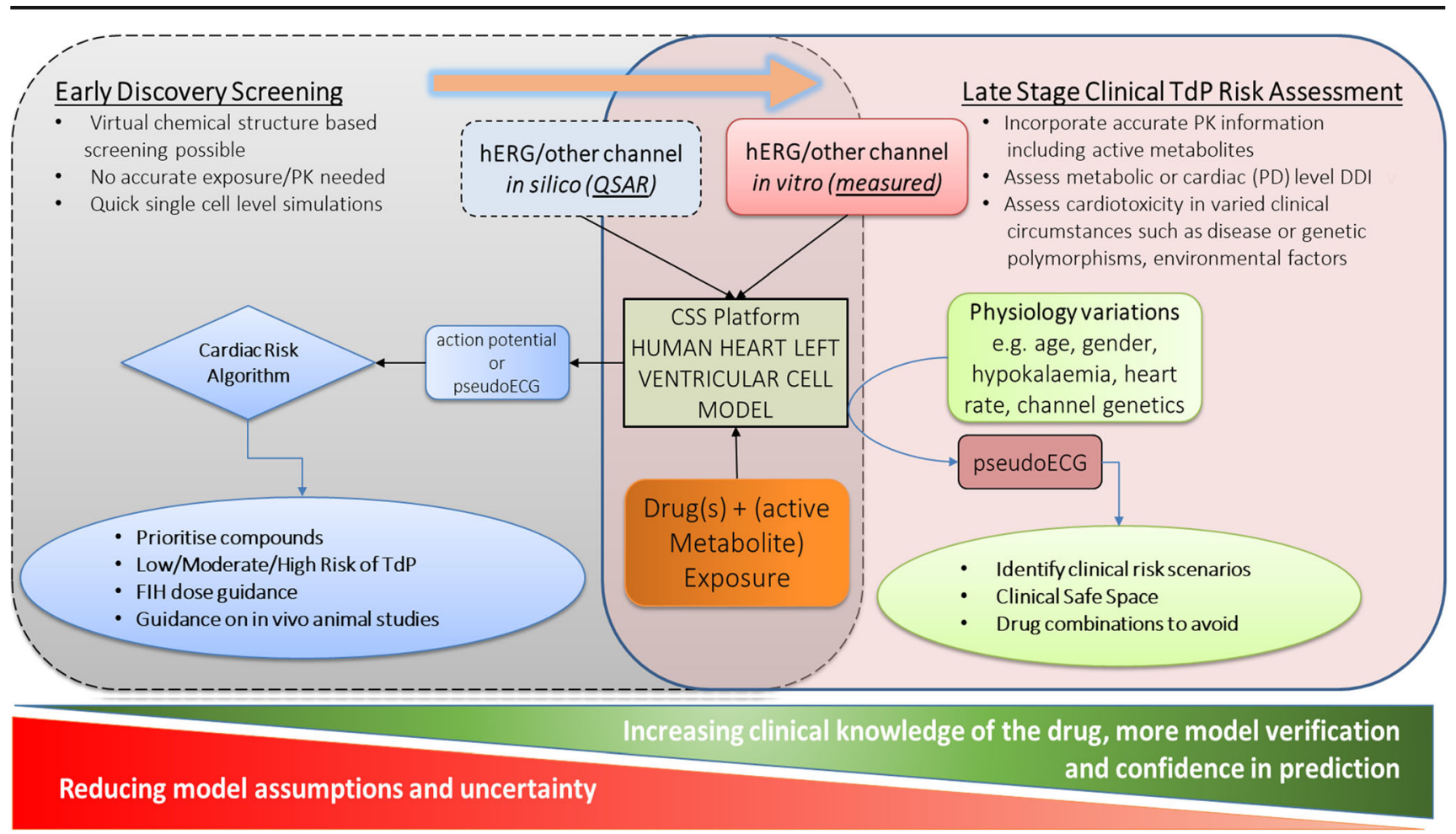

Fig. 1. Progressive cardiac risk assessment strategy in TdP risk assessment

pharmacokinetics of MOXI after oral administration and estimate the population variability in the lung tissue disposition (18), resulting in a mechanistic PK-PD model for translation of the in vitro cardiac safety assessment to clinical level. Poulin and Theil method (19) with Berezhkovskiy correction (20) was used to estimate the tissue:plasma partitioning of MOXI. This method underpredicted the volume of distribution in comparison to volume of distribution reported for MOXI after intravenous administration to human volunteers; hence, the model was calibrated with "Kp scalar" using Simcyp parameter estimation module. The PBPK model was used to derive the concentration-time profiles for various bio-phases used to drive the CSS module (described below). The PBPK model was linked with the mechanistic cardiac QST model for MOXI. As the purpose of the study was to show application of the PBPK-QST framework in early clinical development, a phase 1 healthy volunteer study was simulated. A virtual population of 20 healthy Caucasian individuals (50\% female) aged 20-50 years old was used to simulate single-dose fasted-state administration of a $400-\mathrm{mg}$ MOXI tablet formulation, similar in size to a positive control arm in a typical TQT trial. The resulting plasma concentrationtime profiles were verified by comparison to the observed clinical PK data reported by Stass et al. (21).

The CSS version 2.1 was used to perform all cardiac electrophysiology simulations. The CSS platform combines electro-physiologically based models of the human left ventricular cardiomyocytes and a database of human physiological, genotypic, and demographic data thus allowing generation of a virtual realistic population variability in cardiac physiology during simulations $(13,22)$. Specifically, the CSS platform accounts for circadian variability in heart rate as well as plasma electrolyte $\left(\mathrm{Na}^{+}, \mathrm{K}^{+}\right.$and $\mathrm{Ca}^{2+}$ ) concentrations using covariate models derived from actual clinical data (23). Epidemiological and covariate models which take into account the effect of gender and age on ventricular heart wall thickness, cardiomyocyte volume and capacitance, and sarcoplasmic reticulum volume are also built into the CSS platform for the north European Caucasian population with healthy heart physiology (15). The output from the CSS is in the form of a pseudo-electrocardiogram (i.e., a virtual ECG), including measures of interest (e.g., QRS, QT). It is termed pseudoECG as it is based only on a string of ventricular cells and hence is missing atrial activity characterized by $\mathrm{P}$ wave on a clinical ECG signal.

For this study, the CSS implementation of the ten Tusscher 2006 (TT2006) ventricular cardiomyocyte cell model (24) was used primarily to form a one-dimensional cardiomyocyte string mimicking the ventricular wall cross-section (thickness). The use of another widely used cardiomyocyte model, O'Hara and Rudy (ORd) model (25), was also assessed on the predicted pseudoECG. The cells were paced at the endocardial side and used a 50:30:20 distribution of endocardium, midcardium, and epicardium cells with an average diffusion coefficient of $0.0016 \mathrm{~cm}^{2} / \mathrm{ms}$. The forward Euler method was used to integrate the model equations with a space step and a time step of $0.01 \mathrm{~mm}$ and $0.01 \mathrm{~ms}$, respectively, and a total simulation time of $10,000 \mathrm{~ms}$.

For simulation of the effects of a compound on the pseudoECG outputs, the required model inputs are the in vitro measured $\mathrm{IC}_{50}$ values for relevant cardiac ion currents, operating drug exposure concentrations, time of day (clock time for circadian model), and age and gender of each simulated subject which can be directly read into the CSS platform from the Simcyp PBPK model output. MOXI's cardiac effects are primarily linked to rapid rectifier potassium current $\left(\mathrm{I}_{\mathrm{Kr}}\right)$ inhibition (encoded by hERG gene), as effects on other currents, namely the late calcium $\left(\mathrm{I}_{\mathrm{CaL}}\right)$ and sodium currents $\left(\mathrm{I}_{\mathrm{Na}}\right)$, show $\mathrm{IC}_{50}$ values many-fold higher than the operating physiological tissue concentrations of MOXI after $400 \mathrm{mg}$ single-dose administration and were obtained 
from non-human cell systems. Hence, we considered only the inhibition of $\mathrm{I}_{\mathrm{Kr}}$ by MOXI in our simulations, with corresponding $\mathrm{IC}_{50}$ values obtained from the freely available tox-database.net (Table I) (43). Consistent with other experiments in biological systems, there is considerable inter-laboratory variability in reported $\mathrm{IC}_{50}$ values for $\mathrm{I}_{\mathrm{Kr}}$ inhibition by MOXI, which range from 0.93 to $398 \mu \mathrm{M}$ and are dependent upon in vitro cell line and experimental conditions. Hence, the presumably "most biorelevant" $\mathrm{IC}_{50}$ value of $29 \mu \mathrm{M}$ for $\mathrm{I}_{\mathrm{Kr}}$ inhibition by MOXI as per Alexandrou et al. (26) was chosen an input to the model and a corresponding sensitivity analysis conducted (see Discussion section for further details).

Drug concentration-dependent inhibition of ion channel activity was modeled by multiplying the specified ionic current of interest by an inhibition factor as described in Eq. 1:

Inhibition Factor $=\frac{1}{1+\left(\mathrm{IC}_{50} /[D]\right)^{n}}$

where $\mathrm{IC}_{50}$ is the drug concentration responsible for the $50 \%$ inhibition, $n$ is the shape parameter (Hill coefficient), and $[D]$ indicates the drug concentration in micromolar. The inhibition factor was calculated using MOXI concentrations simulated by the PBPK model for the following bio-phases: (i) unbound plasma concentration, (ii) total plasma concentration, (iii) unbound heart tissue concentration, and (iv) total heart tissue concentration. Each bio-phase concentration was sampled at 13 time points similar to those used in a typical phase 1 study (i.e., 0 , $0.25,0.5,1,1.5,2,2.5,3,4,6,12,18$, and $24 \mathrm{~h}$ post-dose) and used as input to the CSS. The start of the treatment was assumed to be 09:00 o'clock time on day 1 and continues till 09:00 on day 2 $(24 \mathrm{~h})$. The simulated QT interval was corrected for heart rate effect by Fridericia formula (44) and by placebo (QT interval simulated at same time of the day with zero drug concentration) for each individual and each time point. Each of the result sets (corresponding to the four bio-phase concentrations) was then compared with clinically observed QT prolongation reported after single-dose administration of $400 \mathrm{mg}$ orally.

\section{RESULTS}

\section{Performance Verification of PBPK Model}

The simulated plasma concentration-time profile obtained after $400 \mathrm{mg}$ MOXI oral dose in fasted state overlaid with clinically observed data is reported in Fig. 2a. The comparative average unbound plasma, total plasma, unbound heart tissue, and total heart tissue concentrations are shown in Fig. 2b. The concentrations in heart tissue are almost fourfold higher than the corresponding plasma

Table I. MOXI $\mathrm{IC}_{50}$ values for $\mathrm{I}_{\mathrm{Kr}}$ current (marked raw indicates values used in simulations)

\begin{tabular}{|c|c|c|c|c|c|c|c|c|}
\hline $\begin{array}{l}\text { In vitro } \\
\text { cell } \\
\text { model }\end{array}$ & $\begin{array}{l}\text { Temp } \\
\left({ }^{\circ} \mathrm{C}\right)\end{array}$ & $\begin{array}{l}\mathrm{K}^{+} \\
\text {bath } \\
(\mathrm{mM})\end{array}$ & $\begin{array}{l}\text { Holding } \\
\text { potential } \\
(\mathrm{mV})\end{array}$ & $\begin{array}{l}\text { Depolarization } \\
\text { potential }(\mathrm{mV})\end{array}$ & $\begin{array}{l}\text { Repolarization } \\
\text { potential }(\mathrm{mV})\end{array}$ & $\begin{array}{l}\text { Measurement } \\
\text { potential } \\
(\mathrm{mV})\end{array}$ & $\begin{array}{l}\text { Data } \\
\text { source }\end{array}$ & $\begin{array}{l}\text { I C } 50 \\
(\mu \mathrm{M}) \\
(n)\end{array}$ \\
\hline HEK & 22 & 4 & -80 & 20 & -80 & -40 & (26) & $61(0.96)$ \\
\hline HEK & 22 & 4 & -80 & 20 & -80 & -80 & (26) & $65(0.97)$ \\
\hline HEK & 22 & 4 & -80 & - & - & - & (26) & $114(0.94)$ \\
\hline HEK & 35-37 & 4 & -80 & 20 & -80 & -80 & (26) & 29 (1.14) \\
\hline AT-1 & $22-23$ & 4 & -40 & 20 & -40 & -40 & (27) & 0.93 \\
\hline $\mathrm{CHO}$ & $22-24$ & 5.4 & -80 & 20 & -40 & -40 & (28) & $\begin{array}{l}102.63 \\
(1.1)\end{array}$ \\
\hline HEK & $35-36$ & 4 & -80 & 0 & -50 & -50 & (29) & $>100$ \\
\hline HEK & $34-36$ & 4 & -80 & 20 & -80 & -80 & (30) & 35.7 (1) \\
\hline HEK & - & - & - & - & - & - & (31) & 122 \\
\hline $\mathrm{CHO}$ & Room & 5 & -80 & 20 & -40 & -40 & (32) & 129 \\
\hline HEK & Room & 4 & -80 & 40 & -40 & -40 & (33) & $\begin{array}{l}86.2 \\
(0.94)\end{array}$ \\
\hline HEK & $36-38$ & 4 & -75 & 10 & -40 & -40 & (34) & 74.7 \\
\hline HEK & Room & 4 & -80 & 60 & -40 & -40 & (35) & 99 \\
\hline $\mathrm{CHO}$ & 21 & $3.2 ?$ & -70 & 40 & -30 & -30 & (36) & 80.5 \\
\hline HEK & $36.5-37$ & 5 & -80 & 0 & -50 & -50 & (37) & $\begin{array}{l}58.5 \\
(0.92)\end{array}$ \\
\hline $\mathrm{CHO}$ & - & - & -70 & 40 & -30 & -30 & (38) & 398 \\
\hline $\mathrm{CHO}$ & Room & 4 & -80 & 20 & -50 & -50 & (39) & $\begin{array}{l}135 \cdot 8 \\
(0.91)\end{array}$ \\
\hline $\mathrm{CHO}$ & $35-37$ & 4 & -80 & 20 & -40 & -40 & (40) & 34 \\
\hline HEK & - & - & - & - & - & - & (41) & $\left.\begin{array}{lll}1 & 0 & 2 \\
(0.66)\end{array}\right)$ \\
\hline $\mathrm{CHO}$ & $\begin{array}{l}24.5- \\
25.5\end{array}$ & 4 & -80 & 20 & -40 & -40 & (42) & 235 \\
\hline
\end{tabular}

The $\mathrm{IC}_{50}$ value in bold font were considered most bio-relevant and used during QST simulations, see discussion section for further details $H E K$ human embryonic kidney cells, $C H O$ Chinese hamster ovary cells, $A T-1$ atrial tumor myocytes derived from transgenic mice, $I C_{50}$ concentration of drug required to inhibition $50 \%$ of ion channel current, $n$ Hill coefficient 

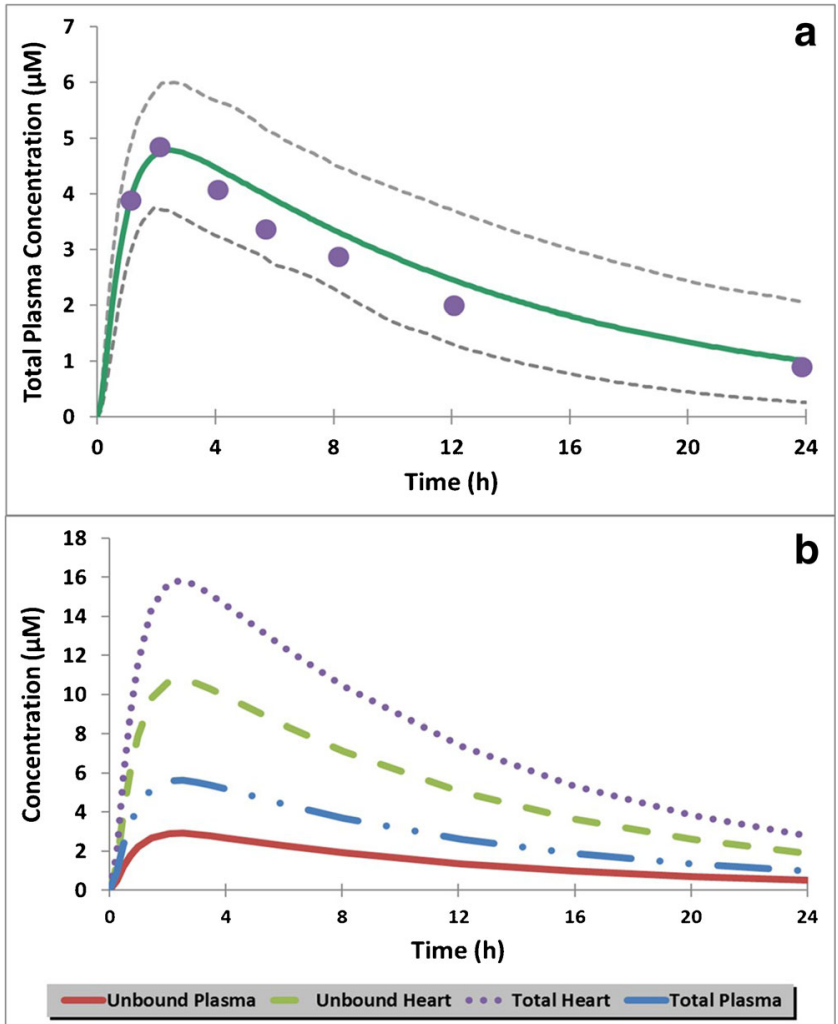

Fig. 2. a Simcyp simulated total plasma concentration time profile (thick green line is arithmetic mean, dotted gray lines are 5th and 95th percentile) from 20 virtual subjects overlaid with mean profile (filled blue circle markers) obtained from clinical study by Stass et al. (21) after 400-mg oral dose of MOXI in fasted state; b Simulated population mean of unbound plasma, total plasma, unbound heart tissue, and total heart tissue concentrations of MOXI

concentrations, and there is a slight delay in achieving $\mathrm{T}_{\max }$ in heart tissue $(2.6 \mathrm{~h})$ as compared to plasma $(2.4 \mathrm{~h})$ due to time taken to perfuse and partition in the heart tissue from systemic circulation.

\section{Performance Verification of the Cardiac QST Model}

Considerable variability in clinical studies measuring QT prolongation is reported in literature mainly due to the differences in study protocols, baseline and heart rate correction approaches, demographic differences, and drug formulations. For example, over encapsulation of MOXI (and the investigational product) is sometimes used for blinding purposes in TQT trials, which may contribute to inter-study variability in MOXI pharmacokinetics, specifically mean transit time and time of peak plasma MOXI concentration as shown by Florian et al. (45). Due to this and other such variations in clinical trial design, before embarking on QST model verification, we summarized the reported QT prolongation from clinical study reports where placebo, baseline and heart rate-corrected QT prolongation, known as $\Delta \Delta \mathrm{QTc}$, was reported. We identified six clinical study reports (46-51) that provide the $\Delta \Delta$ QTc profiles post-MOXI 400-mg oral dose where matching $\mathrm{PK}$ and $\Delta \Delta \mathrm{QTc}$ - time profiles were reported; these are depicted in Fig. 3 along with a pooled average. The profiles were digitized from the graphs reported in study reports using GetData Graph Digitizer software version 2.26 .

The simulated $\Delta \Delta \mathrm{QTcF}$ prolongation derived using simulated MOXI concentrations in the four bio-phases are shown in Fig. 4 and are overlaid with the pooled average $\Delta \Delta$ QTc as shown in Fig. 3. Figure 4a shows the results when unbound plasma MOXI concentration was used as the operating concentration to drive the cardiac QST model, with Fig. 4b-d showing the equivalent for total plasma, unbound heart tissue, and total heart tissue MOXI concentrations, respectively. Comparison of the simulated and observed results clearly shows that the unbound heart tissue concentrations (Fig. 4c) most closely predicted the clinically observed $\Delta \Delta$ QTc values at all the time points.

\section{TdP Risk Prediction at Supra-Therapeutic Exposure and with Other Physiological Risk Factors}

MOXI is considered a potentially torsadogenic drug (52) which is attributed mainly due to its ability to inhibit the hERG channel current. However, the actual reported cases of TdP after MOXI treatment are very rare, which is consistent with the lack of pro-arrhythmic pseudoECG waveforms in any of the virtual patients simulated in Fig. 4 as well as the mid-range probability (54\%) of being TdP positive as estimated by the Cardiac Risk Algorithm of Polak et al. (14). Most of the reported TdP cases occurred when MOXI was administered with other cardiotoxic medications potentiating their TdP effect or physiological conditions and diseases such as hypokalemia, tachycardia, renal failure (main route of elimination for MOXI), or congenital long QT syndrome. Thus, MOXI prolongs the QTc interval at therapeutic dose levels but actual TdP events are contingent upon the presence of other clinical risk factors that may not be present in the healthy volunteer phase 1 trial setting as simulated.

Hence, to simulate a higher-risk clinical situation, the following scenarios were compared with placebo administration in a virtual 20-year-old female subject:

- $\quad$ Scenario 1: MOXI concentrations equivalent to the mean unbound heart tissue MOXI $\mathrm{C}_{\max }$ with 400$\mathrm{mg}$ single-dose administration in a healthy individual; Heart rate 60 beats per min and plasma potassium concentration of $4.2 \mathrm{mM}$

- Scenario 2: Supra-therapeutic MOXI concentrations 10-fold higher than the mean unbound heart tissue MOXI $\mathrm{C}_{\max }$ with 400-mg single-dose administration in a healthy individual;

- Scenario 3: Therapeutic dosing as in scenario 1, but simulated in a virtual patient exhibiting proarrhythmic physiological factors;

Tachycardia (heart rate 120 beats per min) and hypokalemia (plasma $\mathrm{K}^{+}$concentration of $1.5 \mathrm{mM}$ )

- Scenario 4: Supra-therapeutic dosing as in scenario 2 in a virtual patient exhibiting the same proarrhythmic physiological factors as in scenario 3 .

The results of the simulated pseudoECG from the CSS platform are shown in Fig. 5 for all scenarios.

Figure 5a shows that under normal physiological conditions (i.e., scenarios 1 and 2), MOXI prolongs QTc interval without causing any abnormal rhythms even at supra- 


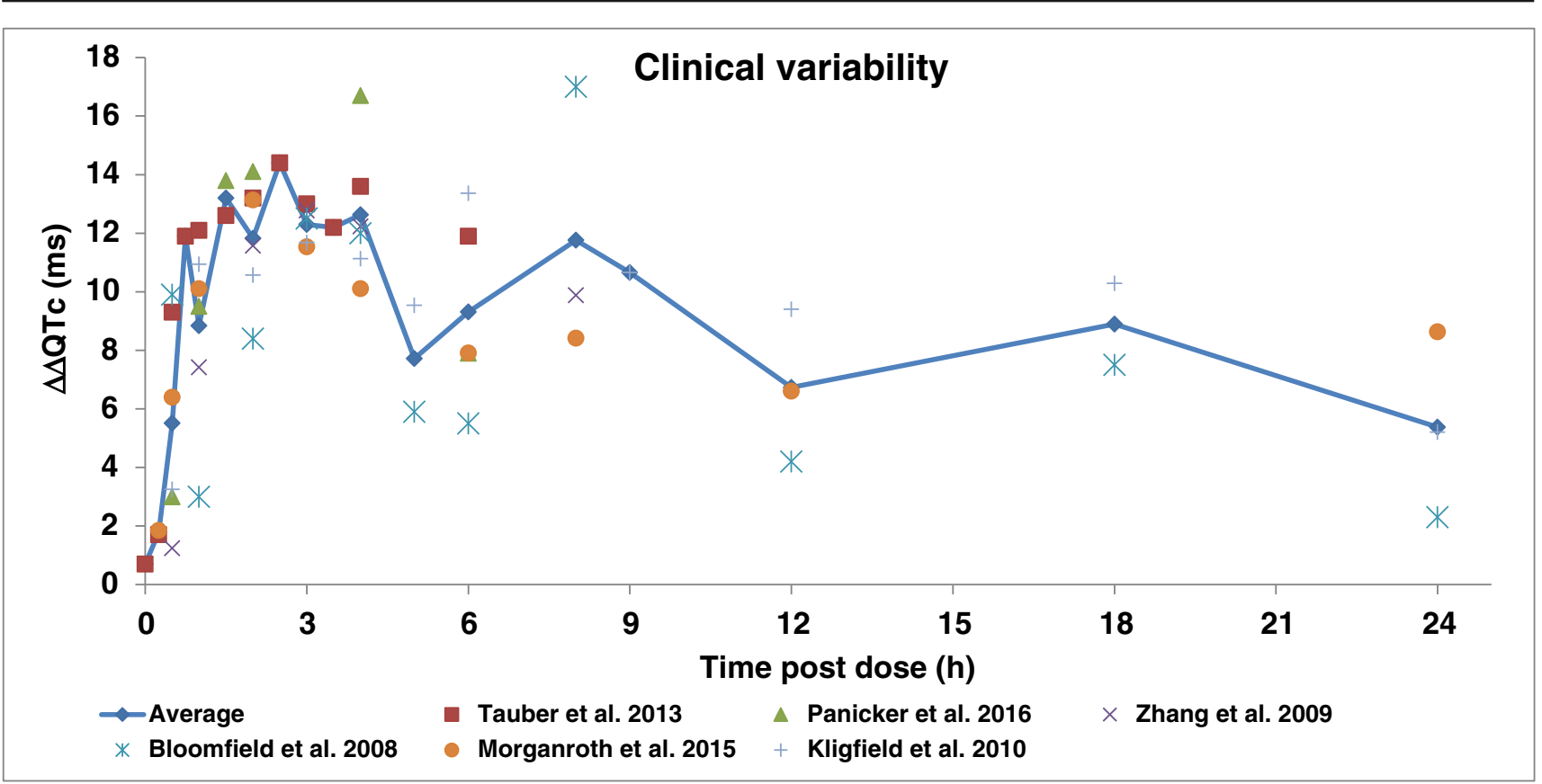

Fig. 3. Variability in clinical $\Delta \Delta$ QTc after 400-mg MOXI oral dose

therapeutic MOXI concentrations. On the other hand, Fig. 5b indicates that in the presence of relevant physiological changes (i.e., hypokalemia and tachycardia), the same supra-therapeutic exposure could cause polymorphic arrhythmia. To put in clinical context, the supra-therapeutic MOXI concentration of $120 \mu \mathrm{M}$ used in scenarios 2 and 4 is 10 -fold higher than the mean unbound heart tissue MOXI $\mathrm{C}_{\max }$ after 400-mg single oral dose administration. These exposures could be seen in a portion of the patient population under various clinical scenarios, such as drug-drug interactions (viz. inhibition of MOXI clearance), overdose or with intravenous bolus administration of high dose of MOXI (>800 mg). Moreover, patients with renal impairment could show higher exposure at therapeutic doses as MOXI is predominantly cleared by renal filtration, which may underlie the reported TdP cases of MOXI in renally impaired patients (53). The results show that MOXI, an otherwise low TdP-risk drug at therapeutic dose in a healthy subject with normal physiology, could cause TdP when combined with elevated patient physiological risk factors.

\section{DISCUSSION}

\section{Predictive Performance of PBPK-QST Model at Therapeutic} and Supra-Therapeutic Exposure

PBPK modeling is widely utilized to estimate drug concentrations in various bio-phases for linking with PD models to establish exposure-effect relationships, without the requirement for a direct measurement of drug concentrations at site of action in clinic $(54,55)$. These models can be semi- or fully mechanistic and can include multiple physiologically relevant pathways. For example, the contributions of transporters in lung uptake of MOXI are incorporated based on in vitro data, which is important in reproducing the observed clinical lung disposition in tuberculosis patients (18). In the cardiac QST framework, heart tissue concentrations have been established as the relevant bio-phase for governing the QT effect rather than concentrations in plasma per in vivo animal work by Minematsu et al. (56). However, given that measurement of heart tissue drug concentrations is not feasible in the clinic due to ethical and practical considerations, suitable concentrations to estimate potential cardiac effects in patients are generally not wellinformed. However, a sufficiently detailed PBPK model that exhibits good predictive performance can potentially help bridge the gap to estimate the concentrations in heart tissue. The robustness of this approach was not fully assessed in the present work, as no clinical or animal data on heart tissue MOXI concentrations was available to verify the PBPK-predicted distribution (i.e., fourfold higher exposure in heart as compared to plasma). Hence, the model predicted heart tissue MOXI concentration and unbound fraction were used "as is" without further refinement as input to the CSS model and drove the pseudoECG output. This approach demonstrated good predictive performance as shown in Fig. 4, where predictions based on the unbound heart tissue MOXI concentration (the most theoretically relevant bio-phase) closely matched the QTc prolongation profile observed in the clinic. Moreover, the CSS model was also able to recover the gender effect in QT prolongation effect, with predicted mean peak $\Delta \Delta$ QTc value $\sim 4.5 \mathrm{~ms}$ higher in females than in male subjects and similar to the $\sim 4 \mathrm{~ms}$ difference reported by Taubel et al. (50). The ability to recapitulate this sex-related difference illustrates an advantage of the model-based QST framework for cardiac risk assessment, as the potential effects of covariates (e.g., food effects, concomitant medications, comorbidities) that affect drug PK and/or PD may be investigated beginning at early development stages. 

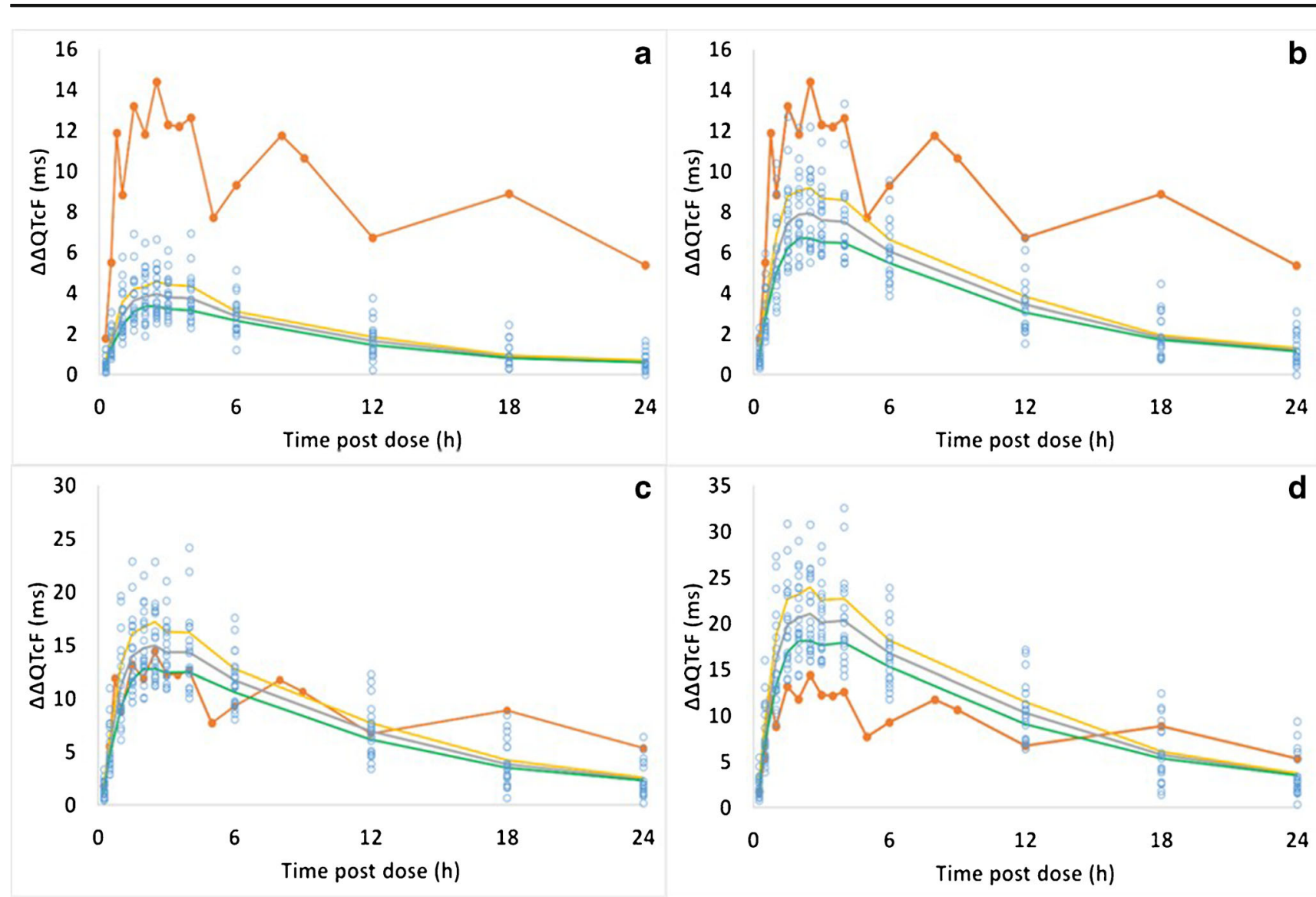

- Sim Individual - Obs Mean -Sim Mean -Sim Female Mean —Sim Male Mean

Fig. 4. TT 2006 model simulated $\Delta \Delta$ QTc-time (post-dose) profile using (a) unbound plasma, (b) total plasma, (c) unbound heart tissue, and (d) total heart tissue concentrations of MOXI as operational concentration to drive the QT response. The population variability of simulated 20 subjects (open circles), average of the population (thick gray line), average of 10 female population group (yellow line), and average of 10 male population group (green line) are also shown for each of simulation set

While the above results indicate that the estimated unbound heart tissue concentration predicted the QT prolongation reasonably well, it is noted that the PBPK model for MOXI is enriched based on non-clinical and clinical PK data to account for discrepancies between "bottom-up" mechanistic predictions of tissue uptake and clinically observed volume of distribution (viz. application of a $\mathrm{Kp}$ scalar). However, simulations such as those presented herein are often conducted during early clinical development, where data is generally more limited, and thus, there is greater uncertainty in the degree of tissue uptake. This uncertainty was reproduced herein as multiple potentially relevant bio-phase concentrations were considered to generate plausible CSS outputs reflecting an approximately fivefold range of mean peak $\Delta \Delta \mathrm{QTc}$ values (i.e., ranging from approximately +4 to $+20 \mathrm{~ms}$ for unbound plasma and total heart MOXI concentrations, respectively). Such an approach is useful in early development, where a set of predictions reflecting a range of potential clinical scenarios may be highly informative in cardiac risk assessment, clinical trial design, and overall clinical development strategy. Even though this work attempted to develop the most mechanistically plausible model based on currently available knowledge in the area, it is worth noting certain uncertainties and assumptions associated with the model and input data. For example, the model did not explicitly account for formulation effects such as over encapsulation, because such information is rarely made publicly available and not well-defined in the clinical studies used for model verification. Secondly, we did not have fully detailed heart tissue model to mimic the exposure inside the cardiomyocyte cell, which has been suggested to be more suitable of exposure for some compounds (57-59). Such detailed heart tissue models exist $(60,61)$, but they could be even more challenging to verify with limited measurements in heart tissue or cardiomyocytes. Moreover, the in vitro $\mathrm{IC}_{50}$ data also reflects the concentration in the media not inside the cell; thus, combining such $\mathrm{IC}_{50}$ without suitable correction with intra-cellular exposure model could produce misleading results. The translatability of in vitro experiments, typically conducted in HEK cells with heterologously expressed cardiac ion channels, to the in vivo situations or actual cardiomyocytes in terms of physiological functions is not fully established. Nonetheless, these are the challenges facing the general cardiotoxicity assessment area, rather than this work specifically, and more experimental data is required to complement such modeling 

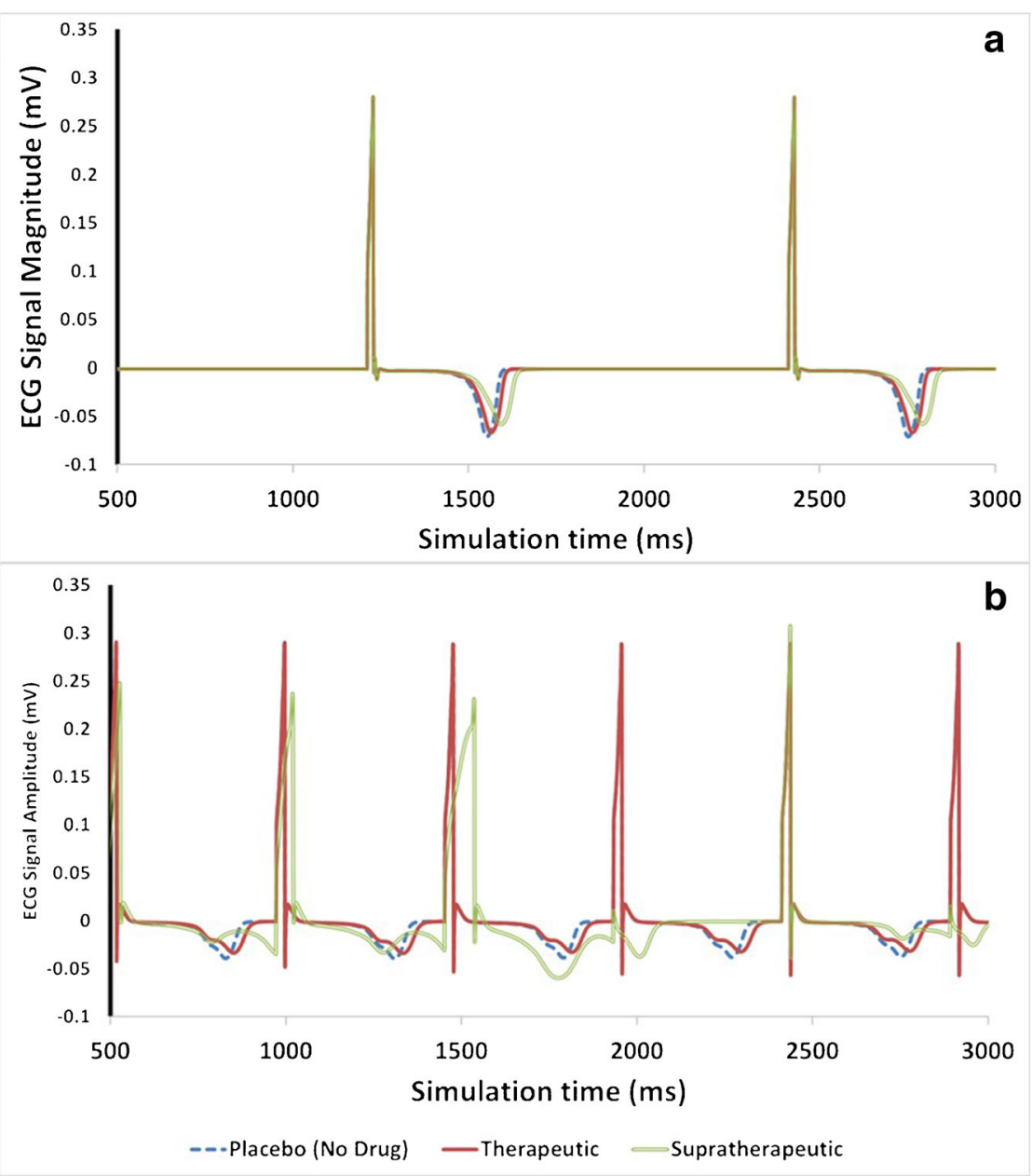

Fig. 5. Simulated pseudoECG traces at therapeutic (red thick line), supra-therapeutic (double green continuous line) exposure of MOXI and placebo (no drug) (dotted blue line) under normal physiological conditions (a), and tachycardia with hypokalaemia (b)

results to test or establish hypotheses and move the field of cardiac risk assessment forward.

Although the estimated unbound heart tissue MOXI concentrations resulted in the best match to the observed clinical data when used as the input for the CSS model, it is noted that PK-PD relationships for cardiac parameters (e.g., QT prolongation) are predominantly reported as correlations with plasma drug concentrations. This correlation is shown in Fig. 6, which depicts the $\Delta \Delta$ QTc values predicted from the CSS using unbound heart tissue MOXI concentration as input versus concentrations at the corresponding time points (unbound heart tissue and total plasma MOXI concentrations in open and closed circles, respectively).

Figure 6 shows robust correlations with the predicted $\Delta \Delta \mathrm{QTcF}$, with slopes of 6.86 and $3.51 \mathrm{~ms} / \mu \mathrm{g} \times \mathrm{mL}^{-1}$ of MOXI in total plasma and unbound heart tissue bio-phases, respectively, with the steeper slope for the plasma correlation reflecting the lower MOXI concentrations in the total plasma vs. the unbound heart tissue bio-phases. Notably, when compared with published slopes from clinical studies, the "bottom-up" predicted slope of $6.86 \mathrm{~ms} / \mu \mathrm{g} \times \mathrm{mL}^{-1}$ for the plasma correlation was similar to slightly higher in magnitude. For example, the value reported by Panicker et al. (49) from a bootstrapping analysis was $5.9 \mathrm{~ms} / \mu \mathrm{g} \times \mathrm{mL}^{-1}$, whereas an earlier population PK-PD study by Florian et al. reported

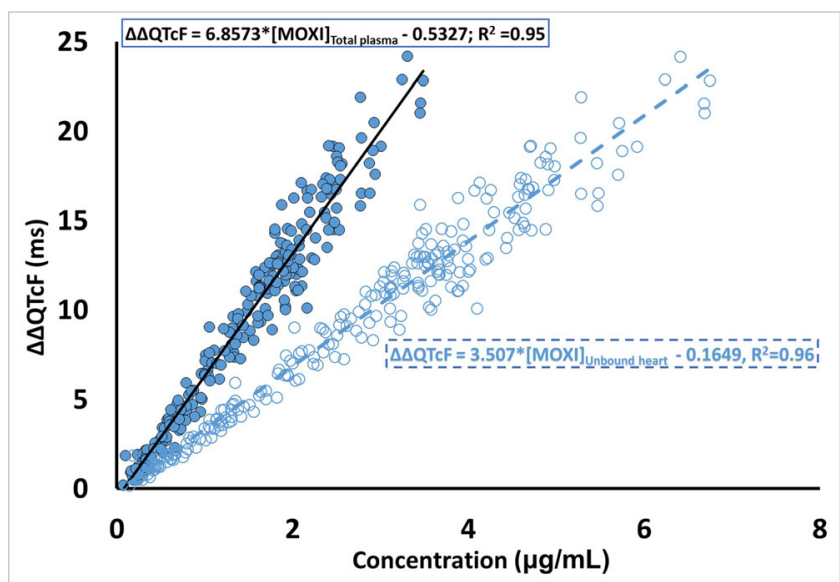

Fig. 6. Relationship between simulated MOXI concentrations and $\Delta \Delta \mathrm{QTcF}$ (filled blue circles represent the total plasma concentration vs. $\Delta \Delta \mathrm{QTcF}$ obtained with unbound heart tissue concentration as operating concentration and open blue circles represent the unbound heart tissue concentration $v s . \Delta \Delta \mathrm{QTcF}$ obtained with unbound heart tissue concentration as operating concentration) 
slopes from individual studies ranging from 1.6 to $4.8 \mathrm{~ms} / \mu \mathrm{g} \times$ $\mathrm{mL}^{-1}$ and a mean estimate of $3.1 \mathrm{~ms} / \mu \mathrm{g} \times \mathrm{mL}^{-1}$ (45). In the latter case, it is noted that this may be influenced by clinical trial size, as clinical studies with larger populations are known to have slopes higher than $3.1 \mathrm{~ms} / \mu \mathrm{g} \times \mathrm{mL}^{-1}$ (62). Regardless, this suggests that the bottom-up approach outlined in the present work provides reasonable, if possibly somewhat conservative, predictions of QTc prolongation for MOXI.

Although the $\Delta \Delta \mathrm{QTcF}$ is a useful comparator for evaluating performance of the PBPK-based CSS simulations, it is important to note that the reliance on QTc as a metric of torsadogenic potential has significant drawbacks $(13,63)$. This is exemplified by MOXI, as reports by Abbasi et al. (64), Okada et al. (39), and simulations done previously by our group (14) have shown with single cell level as well as threedimensional heart wall simulations that MOXI does not cause early after depolarization (EAD) or arrhythmia at up to 100fold supra-therapeutic concentration with healthy cardiac electrophysiology. While this is in contradiction to the reported TdP cases with MOXI in clinic at lower than 100fold exposure levels $(65,66)$, these cases, as well as 274 records ${ }^{1}$ of MOXI-associated TdP (obtained by mining of the US FAERS database using the OpenVigil 2.1 portal (67) for TdP cases reported with MOXI) indicates that almost all cases were multifactorial. Specifically, the MOXI-associated cases involved other contributing factors, such as additional QT prolonging co-medications, disease conditions such as renal failure, or physiology modifications that could influence cardiac electrophysiology such as electrolyte imbalance or abnormal heart rate. It should be emphasized that these findings are highly consistent with pseudoECG simulations presented herein, which also show that MOXI itself is less likely to cause TdP even at 10-fold higher than therapeutic exposure in a healthy subject but could cause arrhythmia when administered to patients with other physiological risk factors or co-medications with torsadogenic potential (refer to Fig. 5). Hence, this demonstrates that the application of the cardiac QST modeling framework shown in this study could potentially help bridge the translational gap by starting with assumption-rich exploratory simulations in early clinical development with iterative refinement through learnconfirm-predict cycles through later stages of drug development. Such an approach is envisioned to guide both appropriate labeling and rational pharmacotherapy of new drugs to avoid or reduce post-marketing TdP risk.

\section{Differences in Reported $I_{\mathbf{r}} I_{C_{50}}$ Values and Its Impact on QST Model Prediction}

Lab to lab variability in cell-based in vitro assays, such as those used to provide input values for use in the present analysis, is well-documented $(43,68)$. This can be seen in the reported $\mathrm{IK}_{\mathrm{r}} \mathrm{IC}_{50}$ values of MOXI from different laboratories and different experimental protocols provided in Table I as well as in the results of an extensive literature search carried out for $\mathrm{IC}_{50}$ values of many drugs and drug-like compounds for various cardiac ion channels (available on tox-

1 Many records are apparent duplicates attributed to reporting by different organizations/individuals; refer to csv file available as the Supplementary Material 2 database.net) (43). However, despite the known variability in lab-based parameters which can potentially strongly influence QST modeling outputs, the impact of this variability is not commonly assessed and reported. To minimize the effects of such variability within the current study, we applied a step-wise strategy to identify the most physiologically relevant experimental input value of $\mathrm{IC}_{50}$ for QST modeling. Results were first triaged based on cell line, where HEK cells were preferred over $\mathrm{CHO}$ cell or other non-human cell lines due to their human origin. Of those based on HEK cells, Alexandrou et al. (26) and Chen et al. (30) used the same experimental protocols, mimicking human physiology with minor difference in operating temperatures. While Lacroix and colleagues (34) have also run their study in the physiological temperature, there were other settings that differed from human physiology (higher value of the holding potential and short pulses) which resulted in their removal from consideration.

Of the remaining two studies, as the experimental conditions used by Alexandrou et al. (26) were the most physiologically relevant, their reported $\mathrm{IC}_{50}$ value of $29 \mu \mathrm{M}$ was used for all the simulation results presented in this publication. However, it is noted that the experimental conditions were very similar differing only in experimental temperature (35-37 vs. $34-36^{\circ} \mathrm{C}$; Alexandrou et al. (26) and Chen et al. (30), respectively). While these differences appear minor, Table I shows that even small temperature changes may have resulted in an approximately $20 \%$ difference in $\mathrm{IC}_{50}$ values, albeit inter-lab, inter-operator, or even inter-run variability may also contribute to the differences. These sources of variability, which seem to be typical for the Patch Clamp technique, may influence decisions taken on the cardiac safety assessment (68). To evaluate the potential impact of the input $\mathrm{IC}_{50}$ value on the model predictions, a sensitivity analysis conducted to study the impact of varying the $\mathrm{IC}_{50}$ from 1 to $300 \mu \mathrm{M}$ on $\Delta \Delta \mathrm{QTcF}$ was conducted (Fig. 7).

An $\mathrm{IC}_{50}$ of around $30 \mu \mathrm{M}$, similar to the 29 - and $35-\mu \mathrm{M}$ values from Alexandrou et al. (26) and Chen et al. (30), gave the predicted $\Delta \Delta \mathrm{QTcF}$ closest to clinically observed. In contrast, higher values as estimated under less physiologically-relevant conditions would have underpredicted the magnitude of QTc

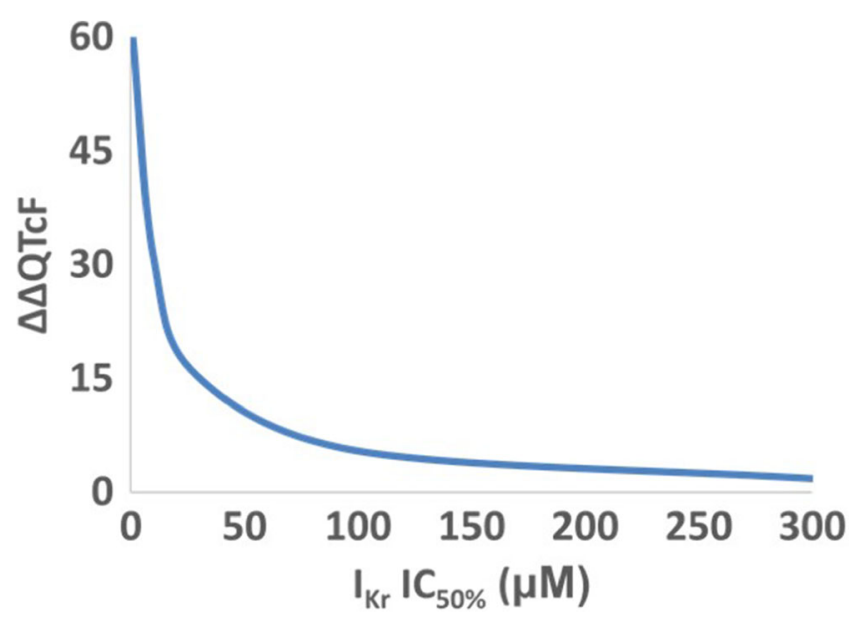

Fig. 7. Sensitivity analysis of predicted $\Delta \Delta \mathrm{QTCF}$ with respect to input $\mathrm{I}_{\mathrm{Kr}} \mathrm{IC}_{50}$ value for MOXI 
prolongation. Thus, it is very important to supply the in vitro input parameters obtained from the experiments conducted at operating conditions mimicking in vivo situations as quality of QST prediction depends heavily on the quality of input information supplied to mechanistic model. It is noted that to aid in translation of $\mathrm{IC}_{50}$ values obtained from different cell lines and different temperatures, the CSS contains validated mathematical inter-system scaling factors (ISSF) (69). However, these factors are only intended to help correct values to better match human physiology, and as such, the ISSF should be used cautiously as they are not intended to replace physiologically relevant in vitro experimentation.

\section{Impact of QST Model Selection on the Results}

The TT2006 model was primarily used to run the pseudoECG simulations in the current work, given the existence of numerous peer-reviewed publications demonstrating their utility in adequately predicting the clinically observed QT prolongation. This model is also relatively fast to run, especially at the pseudoECG level. However, the ORd model and its variants $(70,71)$ have recently been proposed as a method to run single cell simulations to propagate the impact of in vitro $\mathrm{IC}_{50}$ value of ion current inhibition to action potential duration (APD). Although the ORd model to run the pseudoECG simulations offers some additional insights, its overall impact to this work remains limited or negligible, when compared to the use of the TT2006 model. Moreover, the simulations with the ORd model take significantly longer time and show some numerical instability at pseudoECG level in some individuals, when running simulations of unbound plasma and unbound heart tissue concentration exposure. Thorough evaluations of available cardiomyocyte models in terms of the numerical accuracy and their ability to recover observed APD and/or ECG signals are needed to establish the utility of the models to simulate clinical endpoints and/or regulatory use such as CiPA initiative. The impact of model selection on our results is shown in Fig. 8.
The ORd model resulted in larger QT prolongation estimates as compared to the TT2006 model, at the same exposure levels (Figs. 4 and 8). The ORd model showed overand under-estimation of clinically observed QT prolongation profile of MOXI with unbound heart and unbound plasma concentrations, respectively. As such, it is likely that the observed QT prolongation profile is more adequately matched to the total plasma exposure with the ORd model, while the TT2006 model showed best predictions with unbound heart tissue concentrations. As stated above, the aim of the current study was to test physiologically plausible scenarios; therefore, this work does not focus on total plasma concentrations, which would deviate from the hypothesis that only the free drug can be pharmacologically active. Further experimental and in silico work is needed to determine and establish the utility and/or accuracy of various QST models and input data choices to continuously grow the current knowledge base.

\section{CONCLUSIONS}

The assessment of pro-arrhythmic potential and risk of TdP of novel molecules is often challenging given the complex interplay of multiple factors involved requiring a comprehensive understanding of not only the drug-dependent parameters but also the systems characteristics such as human anatomy, physiology, and pathophysiology, as well as external factors, e.g., dosing scheme, concomitantly taken drugs, and co-morbidities. While the method demonstrated herein appears to predict the cardiotoxicity for a well-characterized drug such as MOXI reasonably well, it is envisioned that PBPK-QST modeling be applied throughout development where the component models are refined iteratively through further study per the "learn-confirm-predict-apply" paradigm with enrichment of clinical knowledge of the compound in order to guide decision-making during drug development. Application of a progressive PBPK-QST cardiac risk assessment paradigm starting in early development could guide drug development decisions and later define a clinical "safe
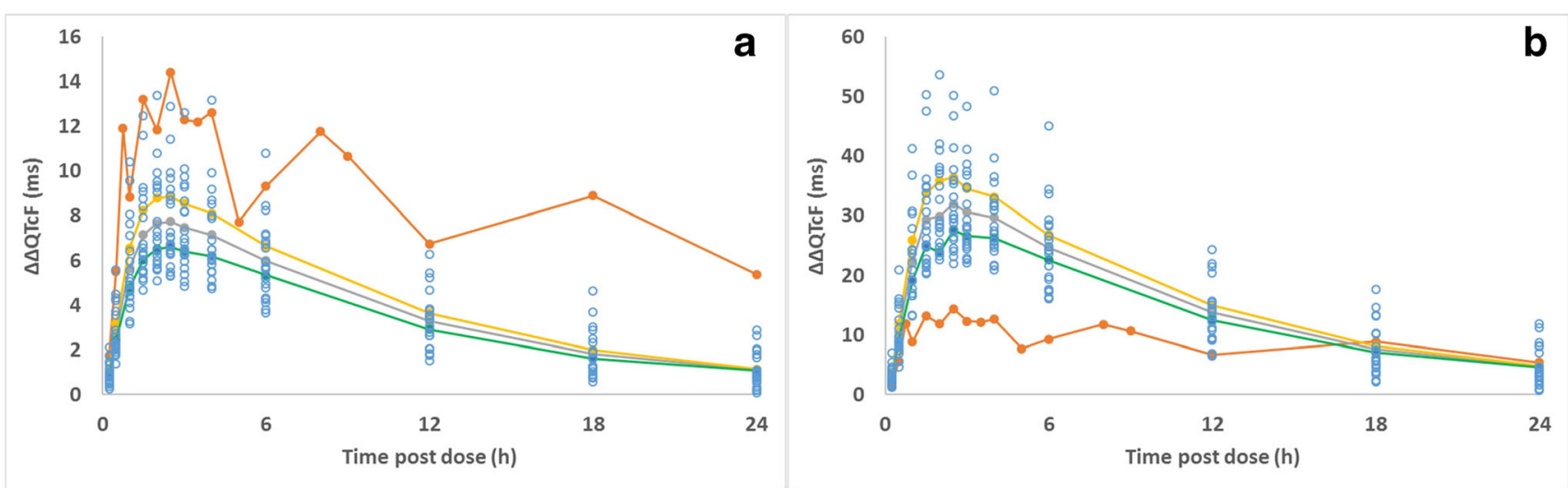

- Sim Individual - Obs Mean Sim Mean

- Sim Female Mean - Sim Male Mean

Fig. 8. ORd model simulated $\Delta \Delta$ QTc-time (post-dose) profile using (a) unbound plasma and (b) unbound heart tissue concentrations of MOXI as operational concentration to drive the QT response. The population variability of simulated 20 subjects (open circles), average of the population (thick gray line), average of 10 female population group (yellow line), and average of 10 male population group (green line) are also shown for each of the simulation set 
space" for post-approval risk management to identify highrisk clinical scenarios. Additional collaborative initiatives are needed to further understand the impact of input data sources and model selections toward establishing good practices to progress the field forward. Further work demonstrating the performance of the QST framework for other drugs and different formulations, routes of administration, co-medications, and diets as well as in the recapitulation of real TdP cases would help to validate this approach and build confidence in proposed progressive risk assessment strategy.

\section{ACKNOWLEDGEMENTS}

Authors would like to thank Lindsay Lehmann and Michael Minchik for proofreading and editorial support in preparation and submission of this manuscript. The Cardiac Safety Simulator and Simcyp Simulator are freely available, following completion of the relevant workshop, to approved members of academic institutions and other non-for-profit organizations for research and teaching purposes.

\section{AUTHOR CONTRIBUTION}

Participated in research design: NP and SP

Conducted Experiments: NP and SP

Performed data analysis: NP

Contributed to the writing of the manuscript: NP, SP, $\mathrm{AB}, \mathrm{KR}, \mathrm{BW}, \mathrm{OH}, \mathrm{DeH}$, andDaH.

\section{COMPLIANCE WITH ETHICAL STANDARDS}

Conflict of Interest NP, SP, and OH are employees of Simcyp Limited (A Certara Company) that provided the CSS and Simcyp Simulator software.

Open Access This article is distributed under the terms of the Creative Commons Attribution 4.0 International License (http://creativecommons.org/licenses/by/4.0/), which permits unrestricted use, distribution, and reproduction in any medium, provided you give appropriate credit to the original author(s) and the source, provide a link to the Creative Commons license, and indicate if changes were made.

\section{REFERENCES}

1. Piccini JP, Whellan DJ, Berridge BR, Finkle JK, Pettit SD, Stockbridge N, et al. Current challenges in the evaluation of cardiac safety during drug development: translational medicine meets the Critical Path Initiative. Am Heart J. 2009;158(3):317-26.

2. Redfern W, Ewart L, Hammond T, Bialecki R, Kinter L, Lindgren $\mathrm{S}$, et al. Impact and frequency of different toxicities throughout the pharmaceutical life cycle. The Toxicologist. 2010;114(S1):1081.

3. Laverty HG, Benson C, Cartwright EJ, Cross MJ, Garland C, Hammond T, et al. How can we improve our understanding of cardiovascular safety liabilities to develop safer medicines? Br J Pharmacol. 2011;163(4):675-93. https://doi.org/10.1111/j.14765381.2011.01255.x.

4. Stockbridge N, Morganroth J, Shah RR, Garnett C. Dealing with global safety issues. Drug Saf. 2013:1-16.
5. Guth BD, Germeyer S, Kolb W, Markert M. Developing a strategy for the nonclinical assessment of proarrhythmic risk of pharmaceuticals due to prolonged ventricular repolarization. J Pharmacol Toxicol Methods. 2004;49(3):159-69.

6. Cavero I, Guillon JM, Ballet V, Clements M, Gerbeau JF, Holzgrefe H. Comprehensive in vitro proarrhythmia assay (CiPA): pending issues for successful validation and implementation. J Pharmacol Toxicol Methods. 2016;81:21-36. https:// doi.org/10.1016/j.vascn.2016.05.012.

7. Colatsky T, Fermini B, Gintant G, Pierson JB, Sager P, Sekino $\mathrm{Y}$, et al. The comprehensive in vitro proarrhythmia assay (CiPA) initiative-update on progress. J Pharmacol Toxicol Methods. 2016;81:15-20. https://doi.org/10.1016/ j.vasen.2016.06.002.

8. Crumb WJ Jr, Vicente J, Johannesen L, Strauss DG. An evaluation of 30 clinical drugs against the comprehensive in vitro proarrhythmia assay (CiPA) proposed ion channel panel. J Pharmacol Toxicol Methods. 2016;81:251-62. https:// doi.org/10.1016/j.vascn.2016.03.009.

9. Fermini B, Hancox JC, Abi-Gerges N, Bridgland-Taylor M, Chaudhary KW, Colatsky T, et al. A new perspective in the field of cardiac safety testing through the comprehensive in vitro Proarrhythmia assay paradigm. J Biomol Screen. 2016;21(1):111. https://doi.org/10.1177/1087057115594589.

10. Vicente J, Zusterzeel R, Johannesen L, Mason J, Sager P, Patel $\mathrm{V}$, et al. Mechanistic model-informed proarrhythmic risk assessment of drugs: review of the "CiPA" Initiative and Design of a Prospective Clinical Validation Study. Clin Pharmacol Ther. 2018;103(1):54-66. https://doi.org/10.1002/cpt.896.

11. Yap YG, Camm AJ. Drug induced QT prolongation and torsades de pointes. Heart. 2003;89(11):1363-72.

12. Patel N, Wiśniowska B, Jamei M, Polak S. Real patient and its virtual twin: application of quantitative systems toxicology modelling in the cardiac safety assessment of citalopram. AAPS J. 2017;20(1):6. https://doi.org/10.1208/s12248-017-0155-8.

13. Polak S, Pugsley MK, Stockbridge N, Garnett C, Wisniowska B. Early drug discovery prediction of proarrhythmia potential and its covariates. AAPS J. 2015;17(4):1025-32. https://doi.org/ 10.1208/s12248-015-9773-1.

14. Polak S, Romero K, Berg A, Patel N, Jamei M, Hermann D, et al. Quantitative approach for cardiac risk assessment and interpretation in tuberculosis drug development J Pharmcokinet Pharcodyn. (2018) Submitted.

15. Polak S, Wisniowska B, Fijorek K, Glinka A, Polak M, Mendyk A, editors. ToxComp+ In vitro-in vivo extrapolation system for drug proarrhythmic potency assessment. Computing in Cardiology (CinC), 2012; 2012: IEEE.

16. Patel N, Polak S, Jamei M, Rostami-Hodjegan A, Turner DB. Quantitative prediction of formulation-specific food effects and their population variability from in vitro data with the physiologically-based ADAM model: a case study using the BCS/BDDCS class II drug nifedipine. Eur J Pharm Sci. 2014;57:240-9. https://doi.org/10.1016/j.ejps.2013.09.006.

17. Jamei M, Turner D, Yang J, Neuhoff S, Polak S, RostamiHodjegan A, et al. Population-based mechanistic prediction of oral drug absorption. AAPS J. 2009;11(2):225-37. https:// doi.org/10.1208/s12248-009-9099-y.

18. Hatley OJ, Patel N, Gaohua L, Burt H, Neuhoff S, Romero K, et al., editors. Application of a multi-compartment permeabilitylimited lung model to predict lung concentrations of moxifloxacin in virtual human subjects. Drug Metabolism Reviews; 2016: Taylor \& Francis Ltd 2-4 Park Square, Milton Park, Abingdon OR14 4RN, Oxon, England.

19. Poulin P, Theil FP. Prediction of pharmacokinetics prior to in vivo studies. 1. Mechanism-based prediction of volume of distribution. J Pharm Sci. 2002;91(1):129-56.

20. Berezhkovskiy LM. Volume of distribution at steady state for a linear pharmacokinetic system with peripheral elimination. J Pharm Sci. 2004;93(6):1628-40. https://doi.org/10.1002/jps.20073.

21. Stass H, Dalhoff A, Kubitza D, Schuhly U. Pharmacokinetics, safety, and tolerability of ascending single doses of moxifloxacin, a new 8-methoxy quinolone, administered to healthy subjects. Antimicrob Agents Chemother. 1998;42(8):2060-5.

22. Polak S, Wisniowska B, Fijorek K, Glinka A, Mendyk A. In vitro-in vivo extrapolation of drug-induced proarrhythmia 
predictions at the population level. Drug Discov Today. 2014;19(3):275-81. https://doi.org/10.1016/j.drudis.2013.10.009.

23. Fijorek K, Puskulluoglu M, Polak S. Circadian models of serum potassium, sodium, and calcium concentrations in healthy individuals and their application to cardiac electrophysiology simulations at individual level. Comput Math Methods Med. 2013;2013:429037. https://doi.org/10.1155/2013/429037.

24. ten Tusscher KH, Panfilov AV. Alternans and spiral breakup in a human ventricular tissue model. Am J Physiol Heart Circ Physiol. 2006;291(3):H1088-100. https://doi.org/10.1152/ ajpheart.00109.2006.

25. O'Hara T, Virag L, Varro A, Rudy Y. Simulation of the undiseased human cardiac ventricular action potential: model formulation and experimental validation. PLoS Comput Biol. 2011;7(5):e1002061. https://doi.org/10.1371/journal.pcbi.1002061.

26. Alexandrou AJ, Duncan RS, Sullivan A, Hancox JC, Leishman DJ, Witchel HJ, et al. Mechanism of hERG K+ channel blockade by the fluoroquinolone antibiotic moxifloxacin. $\mathrm{Br} \mathrm{J}$ Pharmacol. 2006;147(8):905-16. https://doi.org/10.1038/ sj.bjp. 0706678 .

27. Anderson ME, Mazur A, Yang T, Roden DM. Potassium current antagonist properties and proarrhythmic consequences of quinolone antibiotics. J Pharmacol Exp Ther. 2001;296(3):806-10.

28. Bischoff U, Schmidt C, Netzer R, Pongs O. Effects of fluoroquinolones on HERG currents. Eur J Pharmacol. 2000;406(3):341-3.

29. Champeroux P, Ouille A, Martel E, Fowler JS, Maurin A, Richard S, et al. A step towards characterisation of electrophysiological profile of torsadogenic drugs. J Pharmacol Toxicol Methods. 2011;63(3):269-78. https://doi.org/10.1016/ j.vascn.2011.01.001.

30. Chen X, Cass JD, Bradley JA, Dahm CM, Sun Z, Kadyszewski E, et al. QT prolongation and proarrhythmia by moxifloxacin: concordance of preclinical models in relation to clinical outcome. Br J Pharmacol. 2005;146(6):792-9. https://doi.org/ 10.1038/sj.bjp.0706389.

31. Eichenbaum G, Pugsley MK, Gallacher DJ, Towart R, McIntyre $\mathrm{G}$, Shukla U, et al. Role of mixed ion channel effects in the cardiovascular safety assessment of the novel anti-MRSA fluoroquinolone JNJ-Q2. Br J Pharmacol. 2012;166(5):1694707. https://doi.org/10.1111/j.1476-5381.2012.01874.x.

32. Kang J, Wang L, Chen XL, Triggle DJ, Rampe D. Interactions of a series of fluoroquinolone antibacterial drugs with the human cardiac K+ channel HERG. Mol Pharmacol. 2001;59(1):122-6.

33. Kramer J, Obejero-Paz CA, Myatt G, Kuryshev YA, BrueningWright A, Verducci JS, et al. MICE models: superior to the HERG model in predicting torsade de pointes. Sci Rep. 2013;3:2100. https://doi.org/10.1038/srep02100.

34. Lacroix P, Crumb WJ, Durando L, Ciottoli GB. Prulifloxacin: in vitro (HERG current) and in vivo (conscious dog) assessment of cardiac risk. Eur J Pharmacol. 2003;477(1):69-72.

35. Lu HR, Vlaminckx E, Van de Water A, Rohrbacher J, Hermans A, Gallacher DJ. In-vitro experimental models for the risk assessment of antibiotic-induced QT prolongation. Eur J Pharmacol. 2006;553(1-3):229-39. https://doi.org/10.1016/ j.ejphar.2006.09.035

36. Mannikko R, Overend G, Perrey C, Gavaghan CL, Valentin JP, Morten J, et al. Pharmacological and electrophysiological characterization of nine, single nucleotide polymorphisms of the hERGencoded potassium channel. Br J Pharmacol. 2010;159(1):102-14. https://doi.org/10.1111/j.1476-5381.2009.00334.x.

37. Martin RL, McDermott JS, Salmen HJ, Palmatier J, Cox BF, Gintant GA. The utility of hERG and repolarization assays in evaluating delayed cardiac repolarization: influence of multichannel block. J Cardiovasc Pharmacol. 2004;43(3):369-79.

38. Mirams GR, Davies MR, Brough SJ, Bridgland-Taylor MH, Cui Y, Gavaghan DJ, et al. Prediction of thorough QT study results using action potential simulations based on ion channel screens. J Pharmacol Toxicol Methods. 2014;70(3):246-54. https:// doi.org/10.1016/j.vascn.2014.07.002.

39. Okada J, Yoshinaga T, Kurokawa J, Washio T, Furukawa T, Sawada K, et al. Screening system for drug-induced arrhythmogenic risk combining a patch clamp and heart simulator. Sci Adv. 2015;1(4):e1400142. https://doi.org/10.1126/sciadv.1400142.
40. Polonchuk L. Toward a new gold standard for early safety: automated temperature-controlled hERG test on the PatchLiner. Front Pharmacol. 2012;3:3. https://doi.org/10.3389/ fphar.2012.00003.

41. Thomsen MB, Beekman JD, Attevelt NJ, Takahara A, Sugiyama A, Chiba K, et al. No proarrhythmic properties of the antibiotics moxifloxacin or azithromycin in anaesthetized dogs with chronic-AV block. Br J Pharmacol. 2006;149(8):1039-48. https://doi.org/10.1038/sj.bjp.0706900.

42. Yao X, Anderson DL, Ross SA, Lang DG, Desai BZ, Cooper DC, et al. Predicting QT prolongation in humans during early drug development using hERG inhibition and an anaesthetized guinea-pig model. Br J Pharmacol. 2008;154(7):1446-56. https:// doi.org/10.1038/bjp.2008.267.

43. Polak S, Wisniowska B, Glinka A, Polak M. Tox-database.net: a curated resource for data describing chemical triggered in vitro cardiac ion channels inhibition. BMC Pharmacol Toxicol. 2012;13:6. https://doi.org/10.1186/2050-6511-13-6.

44. Fridericia LS. Die Systolendauer im Elektrokardiogramm bei normalen Menschen und bei Herzkranken. J Intern Med. 1920;53(1):469-86.

45. Florian JA, Tornoe CW, Brundage R, Parekh A, Garnett CE. Population pharmacokinetic and concentration-QTc models for moxifloxacin: pooled analysis of 20 thorough QT studies. J Clin Pharmacol. 2011;51(8):1152-62. https://doi.org/10.1177/ 0091270010381498.

46. Bloomfield DM, Kost JT, Ghosh K, Hreniuk D, Hickey LA, Guitierrez MJ, et al. The effect of moxifloxacin on QTc and implications for the design of thorough QT studies. Clin Pharmacol Ther. 2008;84(4):475-80.

47. Kligfield P, Green CL, Mortara J, Sager P, Stockbridge N, Li M, et al. The cardiac safety research consortium electrocardiogram warehouse: thorough QT database specifications and principles of use for algorithm development and testing. Am Heart J. 2010;160(6):1023-8. https://doi.org/10.1016/j.ahj.2010.09.002.

48. Morganroth J, Wang Y, Thorn M, Kumagai Y, Harris S, Stockbridge N, et al. Moxifloxacin-induced QTc interval prolongations in healthy male Japanese and Caucasian volunteers: a direct comparison in a thorough QT study. $\mathrm{Br} \mathrm{J}$ Clin Pharmacol. 2015;80(3):446-59. https://doi.org/10.1111/bcp.12684.

49. Panicker GK, Karnad DR, Kadam P, Badilini F, Damle A, Kothari S. Detecting moxifloxacin-induced QTc prolongation in thorough QT and early clinical phase studies using a highly automated ECG analysis approach. $\mathrm{Br} \mathrm{J}$ Pharmacol. 2016;173(8):1373-80. https://doi.org/10.1111/bph.13436.

50. Taubel J, Ferber G, Lorch U, Batchvarov V, Savelieva I, Camm AJ. Thorough QT study of the effect of oral moxifloxacin on QTc interval in the fed and fasted state in healthy Japanese and Caucasian subjects. Br J Clin Pharmacol. 2014;77(1):170-9. https://doi.org/10.1111/bcp.12168.

51. Zhang X, Silkey M, Schumacher M, Wang L, Raval H, Caulfield JP. Period correction of the QTc of moxifloxacin with multiple predose baseline ECGs is the least variable of 4 methods tested. J Clin Pharmacol. 2009;49(5):534-9. https://doi.org/10.1177/ 0091270008330158.

52. Available from: https://crediblemeds.org/index.php/drugsearch.

53. Altin T, Ozcan O, Turhan S, Ongun Ozdemir A, Akyurek O, Karaoguz R, et al. Torsade de pointes associated with moxifloxacin: a rare but potentially fatal adverse event. Can J Cardiol. 2007;23(11):907-8.

54. Chetty M, Rose RH, Abduljalil K, Patel N, Lu G, Cain T, et al. Applications of linking PBPK and PD models to predict the impact of genotypic variability, formulation differences, differences in target binding capacity and target site drug concentrations on drug responses and variability. Front Pharmacol. 2014;5:258. https://doi.org/10.3389/fphar.2014.00258.

55. Rose RH, Neuhoff S, Abduljalil K, Chetty M, RostamiHodjegan A, Jamei M. Application of a physiologically based pharmacokinetic model to predict OATP1B1-related variability in pharmacodynamics of rosuvastatin. CPT Pharmacometrics Syst Pharmacol. 2014:3:e124. https://doi.org/10.1038/psp.2014.24.

56. Minematsu T, Ohtani H, Yamada Y, Sawada Y, Sato H, Iga T. Quantitative relationship between myocardial concentration of tacrolimus and QT prolongation in guinea pigs: pharmacokinetic/pharmacodynamic model incorporating a site 
of adverse effect. J Pharmacokinet Pharmacodyn. 2001;28(6):533-54.

57. Melgari D, Zhang Y, El Harchi A, Dempsey CE, Hancox JC. Molecular basis of hERG potassium channel blockade by the class Ic antiarrhythmic flecainide. J Mol Cell Cardiol. 2015;86:42-53. https://doi.org/10.1016/j.yjmcc.2015.06.021.

58. Zhang S, Rajamani S, Chen Y, Gong Q, Rong Y, Zhou Z, et al. Cocaine blocks HERG, but not KvLQT1+minK, potassium channels. Mol Pharmacol. 2001;59(5):1069-76.

59. Onohara T, Hisatome I, Kurata Y, Li P, Notsu T, Morikawa K, et al. Molecular mechanisms underlying the pilsicainide-induced stabilization of hERG proteins in transfected mammalian cells. $\mathrm{J}$ Arrhythm. 2017;33(3):226-33. https://doi.org/10.1016/ j.joa.2016.09.003.

60. Tylutki Z, Mendyk A, Polak S. Mechanistic physiologically based pharmacokinetic (PBPK) model of the heart accounting for inter-individual variability: development and performance verification. J Pharm Sci. 2017; https://doi.org/10.1016/ j.xphs.2017.11.012.

61. Tylutki Z, Polak S. A four-compartment PBPK heart model accounting for cardiac metabolism - model development and application. Sci Rep. 2017;7:39494. https://doi.org/10.1038/ srep39494.

62. Shah RR. Drug-induced QT interval prolongation: does ethnicity of the thorough QT study population matter? Br J Clin Pharmacol. 2013;75(2):347-58. https://doi.org/10.1111/j.13652125.2012.04415.x.

63. Sager PT, Gintant G, Turner JR, Pettit S, Stockbridge N. Rechanneling the cardiac proarrhythmia safety paradigm: a meeting report from the cardiac safety research consortium. Am Heart J. 2014;167(3):292-300. https://doi.org/10.1016/ j.ahj.2013.11.004.
64. Abbasi M, Small BG, Patel N, Jamei M, Polak S. Early assessment of proarrhythmic risk of drugs using the in vitro data and single-cell-based in silico models: proof of concept. Toxicol Mech Methods. 2016;27:1-12. https://doi.org/10.1080/ 15376516.2016.1256460.

65. Badshah A, Janjua M, Younas F, Halabi AR, Cotant JF. Moxifloxacin-induced QT prolongation and torsades: an uncommon effect of a common drug. Am J Med Sci. 2009;338(2):164-6. https://doi.org/10.1097/MAJ.0b013e3181a3c2c9.

66. Sherazi S, DiSalle M, Daubert JP, Shah AH. Moxifloxacininduced torsades de pointes. Cardiol J. 2008;15(1):71-3.

67. Bohm R, Hocker J, Cascorbi I, Herdegen T. OpenVigil-free eyeballs on AERS pharmacovigilance data. Nat Biotechnol. 2012;30(2):137-8. https://doi.org/10.1038/nbt.2113.

68. Elkins RC, Davies MR, Brough SJ, Gavaghan DJ, Cui Y, AbiGerges N, et al. Variability in high-throughput ion-channel screening data and consequences for cardiac safety assessment. J Pharmacol Toxicol Methods. 2013;68:112-22.

69. Wisniowska B, Polak S. hERG in vitro interchange factorsdevelopment and verification. Toxicol Mech Methods. 2009;19(4):278-84.

70. Dutta S, Chang KC, Beattie KA, Sheng J, Tran PN, Wu WW, et al. Optimization of an in silico cardiac cell model for proarrhythmia risk assessment. Front Physiol. 2017;8:616. https://doi.org/10.3389/fphys.2017.00616.

71. Li Z, Dutta S, Sheng J, Tran PN, Wu W, Chang K, et al. Improving the in silico assessment of proarrhythmia risk by combining hERG (human ether-a-go-go-related gene) channeldrug binding kinetics and multichannel pharmacology. Circ Arrhythm Electrophysiol. 2017;10(2):e004628. https://doi.org/ 10.1161/CIRCEP.116.004628. 